action of my mind than can be accounted for by experience, further study on many topics, and wider observation of men and things. The most marked change is that memory of the more recent occurrences seems weaker, less accurate, especially in dates, names and sequences of events. My mind recurs to youthful events and dwells on pleasurable or painful matters which then took place, the memory being quite vivid and accurate of things which happened in my teens or the first decade thereafter. I believe this is the experience of most elderly persons. My judgment or reasoning powers do not seem to be impaired with advancing years, though I can not think or conclude as rapidly as in past years, but consume more time in arriving at fixed conclusions."

$\mathrm{Ex}$-Governor Oglesby, thrice governor of Illinois, now 72 years of age, writes me: "I can not say that my mind recurs more frequently to early experiences in advancing age than it did formerly in younger years. As a matter of fact, I have always delighted in reflection upon and remembrances of my childhood duys. I suppose this is quite common with all rational beings."

It is thus evident that the normal dispositions of the mind in advanced age are retrospective and contemplative. Early experiences are the more profound and enduring in their influences on life as "the child is father to the man" and they sink to obscurity by the calls of an ever changing environment of necessity and ambition, until, finally, the pinnacle of experience is reached and historic contemplation begins. The acquisitive faculty wanes with the decline of the sensory and motor powers, and things of recent date become less impressive. Thus, the disposition to retrospection is born of the growing retirement from the daily cares of active combat and the punctuation points of early life which lie at the basis of all character, press forward for the consideration of the wisdom that comes with maturity of decadence as a blessing to rising generations. But the process of degeneration as differing from normal decadence must play a part, though ever so slight, for it is not to be presumed that heredity and environment will leave any individual untainted with the effects of their evils. Also, the highest type of the individual through strain of ambition, may forget the inexorable exactions of nature and thus trespass the conservative limite.

It thus would seem that dotage is not a normal or necessary condition of old age, but a product of degeneration as differing from normal or necessary decline, when at last the servile mechanism of the mind fails to furnish the degree of energy requisite for coöperative labor, time and space cease to be factors in the evolution of the individual and the veil of mystery-the occasion of all effort-remains as ever, to solicit the attention of the living and spur the strong to thought and conquest.

SUMMARY-THREE PRINCIPAL POINTS.

1. That mind is a distinct entity in which there is no heredity, otherwise endless chaos would result. Heredity is exclusively protoplasmic.

2. That for the same reason there can be no disease of mind pure and simple, but that wrong ideas in action necessarily induce disease of the brain, and as one evil begets another, disease of the brain begets wrong ideas, which may become fixed against reason-insanely delusional.
3. That the normal state of mind is self-possession and peace, which is the result of self-sacritice for a. satisfying final purpose.

103 state Street.

\section{THE TREATMENT OF NASAL CATARRH IN THE LONDON CENTRAL THROAT, NOSE AND EAR HOSPITAL.}

BY FAYETTE CLAY EWING, M.D.

Late Senior Clinical Assistant Central Throat, Nose and Ear Hospital and at the London Throat Hospital: Fellow of the British Rhinological, Laryngological and Otological Association, and of the American Medical Association. sT. Louis, Mo.

It occurred to the writer that the treatment of the several forms of nasal catarrh pursued in the Central Hospital would prove interesting to many of the readers of the Journal. ${ }^{1}$ Five varieties are recognized, viz: Acute, chronic, hypertrophic, chronic atrophic, croupous and caseous. "The first of these presents few cases in hospital practice, and the last two are so rare as to be regarded as clinical curiosities.

Acute.-Since patients with this form of rhinitis rarely consult a physician until the acute stage has passed, little is attempted except prophylaxis. This includes abundant open air, exercise under restrictions, and hygienic dress. Light wool next the skin and variable weights of over-garments. Heavy garments indoors are specially cautioned against. No agent is more relied upon for its hardening effect than the morning sponge bath with cold water. This should be taken in a warm room, and supplemented by vigorous rubbing with several towels, the last of which should be a coarse crash. When these rules are observed the most delicate person will suffer noill effects from the bath. Among drugs the first place is given menthol as a prophylactic, and also a modifying agent when the affection is once set up. Cushman's menthol inhaler is recommended to be carried in the pocket, and used frequently during the day as a hardener of the mucous membrane. Menthol is germicidal, and stimulates the capillaries to contraction, thereby relieving obstruction. Cocain, though accomplishing this result much more rapidly, is never prescribed for the purpose, since it produces, after a few applications, dilatation of the vessels, setting up the trouble it originally palliated, and further, there is always danger of the cocain habit being established. During the second stage a mixture composed of camphor, opium and belladonna, in stimulating doses, is advised to be taken between meals.

B. Tr. opii Tr. belladonnæ... Aquæ camphoræ

ài gtt. v. ad 3 i. 30

Misce.

A mixture of menthol and powdered spermaceti, in proportion of 15 grains to the ounce, is frequently used for the same purpose, as a snuff. Quinin is without honor, but Dover's powder is serviceable if the first symptoms of the rhinitis are discernible in the evening.

Chronic Hipertrophic. - The usual symptoms of this disease are nasal obstruction, accompanied by discharge anteriorly and posteriorly. In slight cases where there is very little obstruction a powder is prescribed composed of :

1 Excepting one, the Central Hospital is the largest institution in the world devoted to diseases of the throat, nose and ear, having over 40,000 visits annually. 
R. Potassium chlorat Sodii biborat. Sodii bicarb. Sacch. alb.

pany. Cleanliness is the basis of all treatment. Crusts are immediately removed, and this is followed by a thorough washing with some antiseptic solution such as Dobell's. The removal of crusts is facilitated by the packing of the nasal passages full of cotton wool, which irritates the lining membrane and produces an abundant flow of secretion, loosening the scabs and enabling them to be removed by the forceps. A coarse spray of Dobell's solution follows, the nose-piece fitting tightly in one nostril, and from hand-ball pressure the stream runs in and comes out of the other nostril. When the crusts are adherent to the posterior parts a bent nozzle is passed through the mouth into the post-nasal chamber, and the spray thrown forward. The patient is then ordered to keep the nose clean by using the spray at home. Sometimes the pulv. potass. chlorat. compound is prescribed instead of Dobell's. An oily spray follows the wash the ones commonly advised being:

R. Menthol.

Ol. Olivæ

Misce.

R. Ol. eucalypti Iodol.

Vaselin

Misce.

This treatment is not expected to effect a cure, but is given to render the nose free from odor and keep the patient from being offensive to those with whom he comes in contact. There is another form of treatment of these cases pursued by some of the surgeons connected with this hospital, and believed by them to be absolutely curative. The principle is to create irritation of the mucous membrane sufficient to change the atrophic process. Several plans are followed, but sometimes it is necessary to take the patient into the hospital in order to have the directions carried out. The generally adopted plan is to make the patient, nightly, stuff the nostrils with iodoform wool, after using the spray. One side is packed at a time, the stuffing alternating. $\mathrm{He}$ is made to sleep with it in place if possible. Another way is to paint the entire lining membrane, after cleansing, with a 10 per cent. solution of trichloracetic acid, or with diluted tincture of iodin 1 to 7 . Still another way is to take a small roll of canthos paper, and after the usual washing, a piece of the paper is cut an inch square, and made into a roll and introduced into the nostril, only one side being treated at a time. This is left in place as long as the patient can stand it, which is about twenty minutes. This treatment, and the ones by painting, are repeated every two weeks, but must be prolonged to accomplish good results, and continual spraying is required to prevent relapse. Constitutional treatment is directed in all forms of catarrh, but is considered secondary to local measures. Since the mental state of all victims of atrophic rhinitis demands stimulation, they are encouraged in every way possible and urged to persevere in their treatment.

Caseous.-This is an obstinate and rare affection in which cheesy and putty-like masses block the choanæ. The caseous accumulation fills up the sphenoidal sinus at times and is generally considered as due to caries of the ethmoid bone, but little is positively known of its pathology. The masses are carefully scraped out, and the sphenoidal and ethmoidal cells curetted, when this can be accomplished. The coarse spray is used persistently, and hygienic 
and general constitutional measures prescribed. The disease is regarded as curable if properly and persistently treated.

Croupous.-In its relationship to diphtheritic rhinitis this disease holds a subsidiary position corresponding to that of membranous croup in the larynx. The treatment is rather tedious, since the membrane is very adherent. Alkalin washes are followed by forceps, and an oily spray. Sometimes the nostrils are provided with a lightly carded pledget of menthol wool, 5 to 10 per cent. Tonics and a change of air are often required.

\section{VAGINAL OVARIOTOMY. BY JOHN MADDEN, M.D. MILWAUKEE. WIS.}

Dr. T. G. Thomas, of New York, performed the first systematic vaginal ovariotomy in February of 1870. Before undertaking the operation he prepared himself by operating eight times upon the cadaver. He placed his patient in the knee-elbow position, secured by the apparatus of Bozeman. He passed a rectal bougie into the bowels for a distance of five inches "to prevent all possibility of the rectum falling into the line of incision," lifted up the perineum and posterior wall with a Sims speculum, seized the fornix vaginæ between the rectum and cervix with a tenaculum, and, with a pair of long-handled scissors, made a longitudinal incision into the culde-sac "with one stroke.". The tumor was then seized with a tenaculum, three small cysts ruptured and their contents evacuated, whereupon it was easily drawn into the vagina. The patient was now placed in the dorsal decubitus, the pedicle of tumor ligated with a double-silk ligature, cut off and the stump returned to the abdominal cavity. The incision was closed with a single wire suture.

Dr. Robert Battey performed the operation first in 1874, and in 1877 (Trans. Am. Gyn. Soc.)successful cases were reported by Drs. Gilmore, of Mobile, Clifton E. Wing, of Boston, and Goodell, of Philadelphia. In reporting his case Dr. Goodell mentioned a successful vaginal ovariotomy by Dr. R. Davis, of WilkesBarre, $\mathrm{Pa}$.

In all of the cases here quoted the operation was done for the removal of an enlarged ovary, and are not examples of Battey's operation, which was introduced at this time. After the introduction of Battey's operation, however, a number of operators removed the ovaries per vaginam; but it is not possible to separate the cases belonging to each class.

In Europe vaginal ovariotomy never received any favorable recognition. Whether Hegar, following in the footsteps of Battey, selected the vaginal route for the performance of any of his cases of castration is not recorded. That he did so, is not likely. Olshausen in 1886 (Krank. des Ovar.), remarked that the operation had "never met with favor in Europe and seems to be quite generally abandoned in America, its fatherland." He quotes Mignon of having collected, up to that time, the literature of 113 cases. These cases, however, include all operations in which the ovaries were removed through the vaginal vault, including those in which destructive inflammatory and suppur rative diseases had existed. Since 1887 this mode of operating seems to have been quite completely abandoned; for a careful search through a good deal of gynecologic literature published during the inter- vening eight or nine years failed to discover the record of a single case, and modern text-books dismiss the subject with a half-dozen lines. The complete abandonment of this method of operating is due more, perhaps, to the development of aseptic methods than to any other cause. Fifteen or twenty years ago an abdominal section was not made without hesitation and any method looking to an avoidance of wourding the abdominal peritoneum was looked upon with favor. At the present time the abdominal section has reached a degree of favor little short of an apotheosis. There can scarcely be any doubt, however, that in many cases the vaginal route is preferable to the abdominal. Certainly the operation is too valuable to be cast side.

On the 8th of December last I operated through the vagina for the removal of a cystic ovary, and wish to contribute a description of the operation to the scant literature which now exists upon that subject. The patient was 42 years old, of a nervous temperament, poorly nourished, and gave a history of pelvic disturbance extending over a period of fifteen years. An examination disclosed the presence of a tumor occupying the left side of the pelvis, its lowest part reaching about the level of the internal os. There was no evidence of previous salpingitis, nor attachment of the tumor to surrounding parts; for when the patient was placed in the knee-elbow position the tumor escaped from the pelvic cavity entirely. The pelvis was roomy and the uterus freely movable; but the choice of method was influenced not a little by a lack of faith in being able to have strictly aseptic methods observed in the hospital which the patient had selected. After making the usual preparations, the patient was put in the liththotomy position, the vagina again thoroughly washed out, first with a $1: 1,000$ bichlorid solution, then with sterilized water, and the uterus exposed by means of perineal and vaginal retractors. The lower lip of the uterus was then seized with a pair of strong bulletforceps, drawn down to the vulva and given to an assistant to hold. In the same hand the assistant held the nozzle of a fountain syringe filled with sterilized water, to which had been added bichlorid of mercury, $1: 5,000$, and chlorid of sodium, $6: 1,000$, for continuous irrigation. A transverse incision was now made through the lowest part of the cul-de-sac, close to the uterus. The incision was begun with the scalpel and made large enough, at first, only to allow the insertion of the index finger tip. The bleeding of the afferent uterine vessels was stilled by the introduction of a stitch. The incision was enlarged with a pair of blunt-pointed scissors, a stitch being taken each time to prevent hemorrhage (Fig. 1), be-. fore the tissue was cut. The method adopted is almost exactly like that used by Martin for vaginal extirpation of the uterus, whence this illustration is taken.

After making the incision about $2 \frac{1}{2}$ inches long, the finger was introduced into the pelvic cavity and the tumor found to be entirely free of adhesions. The tumor was now seized with a pair of volcellum for-

1 Since this was written my attention has been called to a case reorted by Staude, of Hamburg Monatssehrift für Geburtshülfe und Gynakologie, Bd. Ir, Heft 4). In this case the tumor was not discovere until labor had begun, when it was felt crowding the posterior wall of the vagina forward. Unable to push it up, Staude opened it, allowing the escape of considerable fluid. He then drew the tumor into the on with such strength and rapidity that he was obliged to finish the operon with such strength and rapidity that he was obliged to finish the opersuture. The patient made an uninterrupted recovery. 